OU-HET 626/2009

\title{
Yukawaon Model in the Quark Sector and Nearly Tribimaximal Neutrino Mixing
}

\author{
Yoshio Koide \\ Department of Physics, Osaka University, Toyonaka, Osaka 560-0043, Japan \\ E-mail address: koide@het.phys.sci.osaka-u.ac.jp
}

\begin{abstract}
For the purpose of deriving the observed nearly tribimaximal neutrino mixing, a possible yukawaon model in the quark sector is investigated. Five observable quantities ( 2 up-quark mass ratios and 3 neutrino mixing parameters $\sin ^{2} 2 \theta_{\text {atm }}, \tan ^{2} \theta_{\text {solar }}$ and $\left.\left|U_{13}\right|\right)$ are excellently fitted by two parameters (one in the up-quark sector and another one in the right-handed Majorana neutrino sector).
\end{abstract}

Keywords: neutrino mixing matrix, CKM mixing matrix, yukawaon model

PACS codes: 12.15.Ff, 11.30.Hv, 12.90.+b, 12.60.Jv

\section{Introduction}

1.1 Why do we investigate a quark mass matrix model for the purpose of discussing the neutrino mixing?

Recently, on the basis of the so-called "yukawaon" model [1, 2] (we will give a short review in the next subsection), the author has proposed a curious neutrino mass matrix form [3]: although the mass matrix $M_{\nu}$ is given by a conventional seesaw-type form $M_{\nu}=m_{D} M_{R}^{-1} m_{D}^{T}$, the Majorana mass matrix $M_{R}$ of the right-handed neutrinos $\nu_{R}$ is related to a up-quark mass matrix $M_{u}$ as

$$
M_{R} \propto M_{u}^{1 / 2} M_{e}+M_{e} M_{u}^{1 / 2},
$$

and the neutrino Dirac mass matrix $m_{D}$ is given by $m_{D} \propto M_{e}$, where $M_{e}$ is the charged lepton mass matrix and $M_{u}^{1 / 2}$ is defined by $\left\langle M_{u}^{1 / 2}\right\rangle_{u}=\operatorname{diag}\left(\sqrt{m_{u}}, \sqrt{m_{c}}, \sqrt{m_{t}}\right)$ on the diagonal basis of $M_{u}$. [Here and hereafter, we denote a form of a matrix $A$ on the diagonal basis of $M_{f}$ (we refer the basis as $f$ basis) as $\langle A\rangle_{f}$.] Therefore, the mass matrix $M_{\nu}$ on the $e$ basis (the diagonal basis of $M_{e}$ ) is given by

$$
\left\langle M_{\nu}\right\rangle_{e} \propto\left\langle M_{e}\right\rangle_{e}\left\{\left\langle M_{u}^{1 / 2}\right\rangle_{e}\left\langle M_{e}\right\rangle_{e}+\left\langle M_{e}\right\rangle_{e}\left\langle M_{u}^{1 / 2}\right\rangle_{e}\right\}^{-1}\left\langle M_{e}\right\rangle_{e}
$$

In order to calculate the lepton mixing matrix $U$, we must to know a form of $\left\langle M_{u}^{1 / 2}\right\rangle_{e}$. Since we do not know it at present, in Ref.[3], on the analogy of a relation $\left\langle M_{u}\right\rangle_{d}=V^{T}(\delta)\left\langle M_{u}\right\rangle_{u} V(\delta)$, where $V(\delta)$ is the Cabibbo-Kobayashi-Maskawa (CKM) quark mixing matrix in the standard expression [4] and the observed value $\delta$ in the quark sector is $\delta \simeq 70^{\circ}$ [5], we have assumed

$$
\left\langle M_{u}^{1 / 2}\right\rangle_{e}=V^{T}(\pi)\left\langle M_{u}^{1 / 2}\right\rangle_{u} V(\pi) .
$$


(Since we assume an $\mathrm{O}(3)$ flavor symmetry, the mass matrices $M_{f}$ must be symmetric, so that the diagonalization is done by $U_{f}^{T} M_{f} U_{f}=M_{f}^{d i a g} \equiv\left\langle M_{f}\right\rangle_{f}$.) Then, by using the observed up-quark mass ratios and CKM mixing parameters, we can obtain the lepton mixing matrix $U$. Usually, the so-called tribimaximal mixing [6] is understood based on discrete symmetries, while, in this model, the neutrino mass matrix (1.2) can give a nearly tribimaximal mixing $\sin ^{2} 2 \theta_{a t m}=0.995$, $\tan ^{2} \theta_{\text {solar }}=0.553$ and $\left|U_{13}\right|=0.001$ [3] without assuming any discrete symmetry. Note that the matrix $V$ in Eq.(1.3) is not $V(\delta)$ with $\delta \simeq 70^{\circ}$, and it must be $V(\pi)$ in order to obtain such the successful results. This successful results rely on the ad hoc assumption (1.3). We have no theoretical ground for the ansatz (1.3).

However, if we give a quark mass matrix model in which quark mass matrices $\left(M_{u}, M_{d}\right)$ is described on the $e$ basis, by using $U_{u}$ obtained from the diagonalization $U_{u}^{T}\left\langle M_{u}\right\rangle_{e} U_{u}=\left\langle M_{u}\right\rangle_{u}$, we can calculate the form of $\left\langle M_{u}^{1 / 2}\right\rangle_{e}$ as $\left\langle M_{u}^{1 / 2}\right\rangle_{e}=U_{u}\left\langle M_{u}^{1 / 2}\right\rangle_{u} U_{u}^{T}$. The purpose of the present paper is to investigate a possible quark mass matrix model which can give reasonable neutrino mixing parameters on the basis of a yukawaon model.

In the present paper, we will propose a quark mass matrix model which is described on the $e$ basis (a diagonal basis of the charged lepton mass matrix):

$$
\begin{aligned}
& \left\langle M_{u}^{1 / 2}\right\rangle_{e} \propto\left\langle M_{e}^{1 / 2}\right\rangle_{e}\left(X+a_{u} \mathbf{1}\right)\left\langle M_{e}^{1 / 2}\right\rangle_{e}, \\
& \left\langle M_{d}\right\rangle_{e} \propto\left\langle M_{e}^{1 / 2}\right\rangle_{e}\left(X+a_{d} e^{i \alpha_{d}} \mathbf{1}\right)\left\langle M_{e}^{1 / 2}\right\rangle_{e},
\end{aligned}
$$

where

$$
X=\frac{1}{3}\left(\begin{array}{lll}
1 & 1 & 1 \\
1 & 1 & 1 \\
1 & 1 & 1
\end{array}\right), \quad \mathbf{1}=\left(\begin{array}{lll}
1 & 0 & 0 \\
0 & 1 & 0 \\
0 & 0 & 1
\end{array}\right)
$$

(Such a form (1.4) has first been proposed in the context of the so-called "democratic seesaw mass matrix model [7].) As we see in the next section, the up-quark mass matrix (1.4) can give not only reasonable up-quark mass ratios at $a_{u} \simeq-0.56$, but also reasonable neutrino mixing parameters $\sin ^{2} 2 \theta_{\text {atm }} \simeq 1, \tan ^{2} \theta_{\text {solar }} \simeq 1 / 2$ and $\left|U_{13}\right| \ll 1$.

\subsection{What is the yukawaon model?}

Prior to investigating a quark mass matrix model, let us give a short review of the socalled yukawaon model: We regard the Yukawa coupling constants in the standard model as "effective" coupling constants $Y_{f}^{e f f}(f=e, \nu, u, d)$ in an effective theory, and we consider that $Y_{f}^{e f f}$ originate in vacuum expectation values (VEVs) of new gauge singlet scalars $Y_{f}$, i.e.

$$
Y_{f}^{e f f}=\frac{y_{f}}{\Lambda}\left\langle Y_{f}\right\rangle
$$

where $\Lambda$ is a scale of the effective theory. We refer the fields $Y_{f}$ as "yukawaons" 1, 2] hereafter. Note that in the yukawaon model, Higgs scalars are the same as ones in the conventional model, i.e. we consider only two Higgs scalars $H_{u}$ and $H_{d}$ as an origin of the masses (not as an origin of the mass spectra). 
The Froggatt-Nielsen model [8] is well known as a model which describes masses and mixings by a VEV value of a scalar $\phi$ : The hierarchical structure of the masses is explained by a multiplicative structure $(\langle\phi\rangle / \Lambda)^{n}$ under a $U(1)$ flavor symmetry. In contrast to the FroggattNielsen model, in the yukawaon model, the scalars $Y_{f}$ have $3 \times 3$ flavor components, so that they are described, for example, as $\mathbf{3} \times \mathbf{3}^{*}=\mathbf{1}+\mathbf{8}$ of $\mathrm{U}(3)$ flavor symmetry or $(\mathbf{3} \times \mathbf{3})_{S}=\mathbf{1}+\mathbf{5}$ of $\mathrm{O}(3)$ flavor symmetry. The hierarchical structures of the quark and lepton masses are understood from hierarchical eigenvalues of $\left\langle Y_{f}\right\rangle$, not from the multiplicative structure $\left(\left\langle Y_{f}\right\rangle / \Lambda\right)^{n}$. In order to obtain such a hierarchical structure, we must build a model with $\operatorname{det}\left\langle Y_{e}\right\rangle /\left(\operatorname{Tr}\left[\left\langle\mathrm{Y}_{\mathrm{e}}\right\rangle\right]^{3}\right) \ll 1$ (for an example, see Ref.[9]). Hierarchical structures of $\left\langle Y_{f}\right\rangle$ in other sectors will be caused by the hierarchical structure of $\left\langle Y_{e}\right\rangle$.

In the present paper, we assume an $\mathrm{O}(3)$ flavor symmetry, so that would-be Yukawa interactions are given by [3]

$$
\begin{gathered}
H_{Y}=\sum_{i, j} \frac{y_{u}}{\Lambda} u_{i}^{c}\left(Y_{u}\right)_{i j} q_{j} H_{u}+\sum_{i, j} \frac{y_{d}}{\Lambda} d_{i}^{c}\left(Y_{d}\right)_{i j} q_{j} H_{d} \\
+\sum_{i, j} \frac{y_{\nu}}{\Lambda} \ell_{i}\left(Y_{\nu}\right)_{i j} \nu_{j}^{c} H_{u}+\sum_{i, j} \frac{y_{e}}{\Lambda} \ell_{i}\left(Y_{e}\right)_{i j} e_{j}^{c} H_{d}+\text { h.c. }+\sum_{i, j} y_{R} \nu_{i}^{c}\left(Y_{R}\right)_{i j} \nu_{j}^{c},
\end{gathered}
$$

where $q$ and $\ell$ are $\mathrm{SU}(2)_{L}$ doublet fields, and $f^{c}(f=u, d, e, \nu)$ are $\mathrm{SU}(2)_{L}$ singlet fields. All of the yukawaons $Y_{f}(f=u, d, \nu, e, R)$ belong to $(\mathbf{3} \times \mathbf{3})_{S}=\mathbf{1}+\mathbf{5}$ of $\mathrm{O}(3)$. In the definition (1.7) of $Y_{f}$, we can define diagonalization of the VEV matrices $\left\langle Y_{f}\right\rangle(f=u, d, e)$ as $U_{f}^{T}\left\langle Y_{f}\right\rangle U_{f} \propto$ $\operatorname{diag}\left(m_{f 1}, m_{f 2}, m_{f 3}\right)$, and that of the seesaw-type neutrino mass matrix $M_{\nu} \propto\left\langle Y_{\nu}\right\rangle^{T}\left\langle Y_{R}\right\rangle^{-1}\left\langle Y_{\nu}\right\rangle$ as $U_{\nu}^{T} M_{\nu} U_{\nu}=\operatorname{diag}\left(m_{\nu 1}, m_{\nu 2}, m_{\nu 3}\right)$, so that we can express the quark mixing matrix $V$ and lepton mixing matrix $U$ as $V=U_{u}^{\dagger} U_{d}$, and $U=U_{e}^{\dagger} U_{\nu}$, respectively. In the interactions (1.7), in order to distinguish each $Y_{f}$ from others, we have assumed a $\mathrm{U}(1)_{X}$ symmetry in addition to the $\mathrm{O}(3)$ flavor symmetry, and we have assigned $\mathrm{U}(1)_{X}$ charges ("sector" charges, not "flavor" charges) as $Q_{X}\left(f^{c}\right)=-x_{f}, Q_{X}\left(Y_{f}\right)=+x_{f}$ and $Q_{X}\left(Y_{R}\right)=2 x_{\nu}$. The $\mathrm{SU}(2)_{L}$ doublet fields $q$, $\ell, H_{u}$ and $H_{d}$ are assigned as sector charges $Q_{X}=0$. (For the right-handed neutrinos $\nu^{c}$, we assume $Q_{X}\left(\nu^{c}\right)=Q_{X}\left(e^{c}\right)$, so that the yukawaon $Y_{e}$ can couple to the Dirac neutrino sector $\ell_{i} \nu_{j}^{c}$ as well as $\ell_{i} e_{j}^{c}$, and we can build a model without $Y_{\nu}$ [10].)

The $\mathrm{O}(3)$ flavor symmetry is broken at the energy scale $\mu=\Lambda$, so that all the yukawaons $Y_{f}$ have VEVs with the order of $\mu=\Lambda$. (We consider that all components $v_{f i}$ of $\left\langle Y_{f}\right\rangle_{f}=$ $\operatorname{diag}\left(v_{f 1}, v_{f 2}, v_{f 3}\right)$ simultaneously have non-zero values at the same energy scale $\Lambda$ in spite of the hierarchical structure $\left|v_{f 3}\right|^{2} \gg\left|v_{f 2}\right|^{2} \gg\left|v_{f 1}\right|^{2}$.) A naive estimate of $\Lambda$ leads to $m_{\nu} \sim\left\langle H_{u}^{0}\right\rangle^{2} / \Lambda$, so that we consider $\mu=\Lambda \sim 10^{15} \mathrm{GeV}$. Since the $\mathrm{O}(3)$ flavor symmetry is completely broken at $\mu=\Lambda$, the effective coupling constants $Y_{f}^{e f f}=\left(y_{f} / \Lambda\right)\left\langle Y_{f}\right\rangle$ evolve as in the standard model below the scale $\Lambda$.

In a supersymmetric (SUSY) yukawaon model, VEV structures of the yukawaons are obtained from SUSY vacuum conditions for a superpotential $W$. As a result, a VEV structure of $\left\langle Y_{f}\right\rangle$ in an $f$ sector is described in terms of other yukawaon VEV matrices. In other words, a VEV structure of $\left\langle Y_{f}\right\rangle$ is given by observed mass matrices in other sectors. (The relation (1.1) is one of the examples.) This is just a characteristic feature in the yukawaon approach. 
For example, in the present scenario, we have assumed superpotential terms

$$
W_{e}=\mu_{e}\left[Y_{e} \Theta_{e}\right]+\lambda_{e}\left[\Phi_{e} \Phi_{e} \Theta_{e}\right]
$$

and, from the SUSY vacuum coditions $\partial W / \partial \Theta_{e}=0$, we obtain a bilinear mass relation

$$
\left\langle Y_{e}\right\rangle=-\frac{\lambda_{e}}{\mu_{e}}\left\langle\Phi_{e}\right\rangle\left\langle\Phi_{e}\right\rangle
$$

Thus, the mass matrix $M_{e}^{1 / 2}$ in Eq.(1.2) is interpreted as $\left\langle\Phi_{e}\right\rangle$ in the yukawaon model. We call $\Phi_{e}$ as "ur-yukawaon", because the VEV of $\Phi_{e}$ plays a role in giving VEV spectrum of $Y_{e}$. Here and hereafter, for convenience, we denote $\operatorname{Tr}[A]$ as $[A]$ simply. (We can show $\left\langle\Theta_{e}\right\rangle=0$ by solving SUSY vacuum conditions for all fields simultaneously. Hereafter, we will use a character $\Theta$ for a field whose VEV is $\langle\Theta\rangle=0$.) Also, we consider the existence of an "ur-yukawaon" $\Phi_{u}$ which has a relation $\left\langle Y_{u}\right\rangle \propto\left\langle\Phi_{u}\right\rangle\left\langle\Phi_{u}\right\rangle$ similar to (1.8).

The relation (1.1) is naively translated to a relation $\left\langle Y_{R}\right\rangle \propto\left\langle\Phi_{u}\right\rangle\left\langle Y_{e}\right\rangle+\left\langle Y_{e}\right\rangle\left\langle\Phi_{u}\right\rangle$ in the yukawaon model, but, as stated in the next section, the relation (1.1) will be slightly modified (a term with same $\mathrm{U}(1)_{X}$ charge will be added with an additional parameter $\xi$ ). As a result, as we see in Sec.2, we can excellently fit 5 observable quantities ( 2 up-quark mass ratios and 3 neutrino mixing parameters) by the two parameters $a_{u}$ and $\xi$. Considering such the phenomenological success of the model, in Sec.3, a possible yukawaon model in the quark sector and neutrino sector is discussed. Finally, Sec.4 is devoted to a summary and concluding remarks.

In the present model, 2 down-quark mass ratios and 4 CKM mixing parameters are described by the remaining 2 parameters $a_{d}$ and $\alpha_{d}$ after $a_{u}$ was fixed by the observed up-quark mass ratios. As seen in Sec.2, the results are still unsatisfactory. We need a further improvement for downquark sector. In the present paper, we do not discuss any improvement in the down-quark sector.

\section{Phenomenological study}

In the present section, we give numerical studies based on phenomenological mass matrices (1.2) and (1.4). Since the aim of the present paper is not to obtain the best fit values of the parameters in the model, and it is to see a main framework of the model qualitatively, an energy scale used in the present evaluations is not required to be so rigorous. Exactly speaking, VEV relations in the present model are valid at $\mu=\Lambda \sim 10^{15} \mathrm{GeV}$. Since the $\mathrm{O}(3)$ flavor symmetry is completely broken at $\mu=\Lambda$, the effective coupling constants $Y_{f}^{\text {eff }}=\left(y_{f} / \Lambda\right)\left\langle Y_{f}\right\rangle$ evolve as in the standard model below the scale $\Lambda$. We know that the quark mass ratios are not so sensitive to the energy scale [11. As seen in (2.1) and (2.6) below, the observed quark mass values [11] still have large errors and, besides, they are highly dependent on the value of $\tan \beta$ in the SUSY model. Since our concern is in the quark mixing matrix $V=U_{u}^{\dagger} U_{d}$ and lepton mixing matrix $U=U_{e}^{\dagger} U_{\nu}$, for simplicity, we evaluate the mass matrices (1.4) at the energy scale $\mu=m_{Z}$, so that numerical results in the present section should not be taken too strictly. In the present model, quark mass matrices are described in terms of the charged lepton masses, so that, for numerical estimates, we will also use values of the running masses $m_{e}(\mu), m_{\mu}(\mu)$ and $m_{\tau}(\mu)$ at 
$\mu=m_{Z}$. Although the yukawaon model [2] was first proposed with the aim of understanding the well-known charged lepton mass relation [12], in the present paper, we do not adopt such a yukawaon model in the charged lepton sector. We will use the observed values [11] of the running charged lepton masses as input values, which do not satisfy the charged lepton mass relation.

First, we search for a value of the parameter $a_{u}$ which can give reasonable predicted values for the up-quark mass ratios [11] $m_{u} / m_{c}=0.0021_{-0.0008}^{+0.0013}\left(0.0021_{-0.0009}^{+0.0012}\right)$ and $m_{c} / m_{t}=$ $0.0036_{-0.0005}^{+0.0006}\left(0.0026_{-0.0006}^{+0.0007}\right)$ at $\mu=m_{Z}\left(\right.$ at $\mu=\Lambda_{G U T}=2 \times 10^{16} \mathrm{GeV}$ with $\left.\tan \beta=10\right)$. Although the ratio $m_{u} / m_{t}$ is affected by renormalization group equation (RGE) effects, the effects are not so essential in this rough estimations in the present paper. Therefore, we will use values at $\mu=m_{Z}$ :

$$
\sqrt{m_{u} / m_{c}}=0.045_{-0.010}^{+0.013}, \quad \sqrt{m_{c} / m_{t}}=0.060 \pm 0.005 .
$$

We find that predicted values of the up-quark mass ratios at $a_{u} \simeq-0.56$ is in favor of the observed up-quark mass ratios (2.1): $v_{u 1} / v_{u 2}=(-0.0355,-0.0425,-0.0514)$ and $v_{u 2} / v_{u 3}=$ $(-0.0654,-0.0570,-0.0495)$ for $a_{u}=(-0.55,-0.56,-0.57)$. However, if we naively regard $\left\langle\Phi_{u}\right\rangle_{e}$ given in Eq.(1.4) as $\left\langle M_{u}^{1 / 2}\right\rangle_{e}$ in Eq.(1.2), we cannot obtain favorable values of the neutrino mixing parameters for any values of $a_{u}$.,

Note that signs of the eigenvalues $\left(v_{u 1}, v_{u 2}, v_{u 3}\right)$ of $\left\langle\Phi_{u}\right\rangle$ are $(+,-,+)$ for the parameter value $a_{u} \sim-0.56$, while, in Ref. [3], we have used positive values for $\left\langle M_{u}^{1 / 2}\right\rangle_{e}=\operatorname{diag}\left(\sqrt{m_{u}}, \sqrt{m_{c}}, \sqrt{m_{t}}\right)$. Therefore, by introducing an $\mathrm{O}(3) \mathbf{1}+\mathbf{5}$ field $P_{u}$ whose VEV is given by

$$
\left\langle P_{u}\right\rangle_{u}=\mu_{p} \operatorname{diag}(+1,-1,+1),
$$

we can express $\left\langle M_{u}^{1 / 2}\right\rangle$ as $\left.\left\langle M_{u}^{1 / 2}\right\rangle_{u} \propto \Phi_{u}\right\rangle_{u}\left\langle P_{u}\right\rangle_{u}$, so that the relation (1.2) is given by

$$
\left\langle Y_{R}\right\rangle_{e} \propto\left\langle Y_{e}\right\rangle_{e}\left\langle P_{u}\right\rangle_{e}\left\langle\Phi_{u}\right\rangle_{e}+\left\langle\Phi_{u}\right\rangle_{e}\left\langle P_{u}\right\rangle_{e}\left\langle Y_{e}\right\rangle_{e}
$$

where $\left\langle\Phi_{u}\right\rangle_{e}$ and $\left\langle P_{u}\right\rangle_{e}$ are given by

$$
\left\langle\Phi_{u}\right\rangle_{e}=U_{u}\left\langle\Phi_{u}\right\rangle_{u} U_{u}^{T}, \quad\left\langle P_{u}\right\rangle_{e}=U_{u}\left\langle P_{u}\right\rangle_{u} U_{u}^{T}
$$

respectively. However, when we assign $\mathrm{U}(1)_{X}$ charges which satisfy $Q_{X}\left(Y_{R}\right)=Q_{X}\left(Y_{e}\right)+$ $Q_{X}\left(\Phi_{u}\right)+Q_{X}\left(P_{u}\right)$, we must also take terms $P_{u} Y_{e} \Phi_{u}+\Phi_{u} Y_{e} P_{u}$ into consideration as well as the terms $Y_{e} P_{u} \Phi_{u}+\Phi_{u} P_{u} Y_{e}$, because they have the same $\mathrm{U}(1)_{X}$ charges. Thus, the relation (2.3) must be modified as

$$
Y_{R} \propto Y_{e} P_{u} \Phi_{u}+\Phi_{u} P_{u} Y_{e}+\xi\left(P_{u} Y_{e} \Phi_{u}+\Phi_{u} Y_{e} P_{u}\right)
$$

We find that predicted value of $\tan ^{2} \theta_{\text {solar }}$ is highly dependent on the parameter $\xi$, although the values $\sin ^{2} 2 \theta_{a t m}$ and $\left|U_{13}\right|$ are not sensitive to $\xi$ as far as $|\xi|$ is small. We show $\xi$-dependence of the neutrino mixing parameters in Table 1 . As seen in Table 1 , the cases $\xi=+0.0005$ and $\xi=-0.0012$ can excellently fit the observed values $\sin ^{2} 2 \theta_{\text {atm }}=1.00_{-0.13}$ [13] and $\tan ^{2} \theta_{\text {solar }}=$ $0.469_{-0.041}^{+0.047}$ [14. At present, the reason why the parameter value of $\xi$ is so small is unknown. This may be explain by a hidden symmetry which is unbroken in the terms without $\xi$, but is broken in those with $\xi$. This is an open question at present. 
Table 1: $\xi$-dependence of neutrino mixing parameters. The parameter $a_{u}$ is fixed at $a_{u} \sim-0.56$.

\begin{tabular}{|c|ccc|}
\hline$\xi$ & $\sin ^{2} 2 \theta_{\text {atm }}$ & $\tan ^{2} \theta_{\text {solar }}$ & $\left|U_{13}\right|$ \\
\hline 0 & 0.9848 & 0.7033 & 0.0128 \\
\hline+0.004 & 0.9825 & 0.4891 & 0.0123 \\
+0.005 & 0.9819 & 0.4486 & 0.0122 \\
+0.006 & 0.9812 & 0.4123 & 0.0120 \\
\hline-0.0011 & 0.9897 & 0.4854 & 0.0142 \\
-0.0012 & 0.9900 & 0.4408 & 0.0143 \\
-0.0013 & 0.9904 & 0.4008 & 0.0144 \\
\hline
\end{tabular}

Table 2: CKM mixing parameters versus $\left(a_{d}, \alpha_{d}\right)$

\begin{tabular}{|c|cc|cc|cccc|}
\hline$a_{u}$ & $a_{d}$ & $\alpha_{d}$ & $\left|m_{d 1} / m_{d 2}\right|$ & $\left|m_{d 2} / m_{d 3}\right|$ & $\left|V_{u s}\right|$ & $\left|V_{c b}\right|$ & $\left|V_{u b}\right|$ & $\left|V_{t d}\right|$ \\
\hline-0.56 & -0.620 & $4^{\circ}$ & 0.1078 & 0.0273 & 0.2035 & 0.0666 & 0.0101 & 0.0178 \\
-0.56 & -0.625 & $6^{\circ}$ & 0.0783 & 0.0313 & 0.2187 & 0.0818 & 0.0123 & 0.0190 \\
-0.56 & -0.630 & $8^{\circ}$ & 0.0542 & 0.0362 & 0.2222 & 0.0977 & 0.0146 & 0.0194 \\
-0.58 & -0.630 & $2^{\circ}$ & 0.1959 & 0.0195 & 0.2272 & 0.0448 & 0.0088 & 0.0163 \\
\hline
\end{tabular}

Next, we calculate down-quark mass ratios and CKM matrix parameters for the model (1.4). The observed running down-quark mass ratios [11] are

$$
\frac{m_{d}}{m_{s}}=\begin{aligned}
& 0.053_{-0.029}^{+0.051} \\
& \left(0.054_{-0.030}^{+0.058}\right)
\end{aligned}, \quad \frac{m_{s}}{m_{b}}=\begin{gathered}
0.019 \pm 0.006 \\
\left(0.017_{-0.005}^{+0.006}\right)
\end{gathered}
$$

at $\mu=m_{Z}$ (at $\mu=\Lambda_{G U T}=2 \times 10^{16} \mathrm{GeV}$ with $\tan \beta=10$ ) and the observed CKM mixing parameters [5] are $\left|V_{u s}\right|=0.2255 \pm 0.0019,\left|V_{c b}\right|=0.0412 \pm 0.0011,\left|V_{u b}\right|=0.00393 \pm 0.00036$ and $\left|V_{t d}\right|=0.0081 \pm 0.025$. In Table 2 , we demonstrate predicted values of the CKM mixing parameters versus $\left(a_{d}, \alpha_{d}\right)$. Here, we have taken $a_{u}=-0.56$ which can give reasonable up-quark mass ratios.

As seen in Table 2 , the case with $a_{u}=-0.56$ and $\left(a_{d}, \alpha_{d}\right)=\left(-0.63,8^{\circ}\right)$ can roughly give reasonable values of the down-quark mass ratios and $\left|V_{u s}\right|$, but $\left|V_{c b}\right|,\left|V_{u b}\right|$ and $\left|V_{t d}\right|$ are considerably larger than the observed values. For reference, we list a case of $a_{u}=-0.58$ and $\left(a_{d}, \alpha_{d}\right)=\left(-0.63,2^{\circ}\right)$ in Table 3 . The case can give reasonable values of $\left|V_{u s}\right|$ and $\left|V_{c b}\right|$, but the predicted quark mass ratios are in poor agreement with experiments. The present model will need a further improvement, as far as the down-quark sector is concerned. However, from the qualitative point of view, it worthwhile noticing that the model can roughly predict not only the quark mass ratios but also the CKM mixing parameters and neutrino oscillation parameters by using only the 4 input parameters $a_{u}, \xi$ and $a_{d} e^{i \alpha_{d}}$.

\section{Yukawaons in the quark sector}

In the previous section, we have obtained successful predictions on the basis of the phe- 
nomenological mass matrices (1.4) and (2.5). For the phenomenological mass matrix (2.5), we assume the following superpotential terms

$$
W_{R}=\mu_{R}\left[Y_{R} \Theta_{R}\right]+\frac{\lambda_{R}}{\Lambda}\left\{\left[\left(Y_{e} P_{u} \Phi_{u}+\Phi_{u} P_{u} Y_{e}\right) \Theta_{R}\right]+\xi\left[\left(P_{u} Y_{e} \Phi_{u}+\Phi_{u} Y_{e} P_{u}\right) \Theta_{R}\right]\right\}
$$

Here, since the VEV matrix $\left\langle P_{u}\right\rangle$ is diagonal in the $u$ basis (a diagonal basis of $\left\langle Y_{u}\right\rangle$ ) as given in Eq.(2.2), the VEV matrix $\left\langle P_{u}\right\rangle$ must be commutable with $\left\langle\Phi_{u}\right\rangle$. Moreover, in order to give the form $(2.2)$, since $\left[\left\langle P_{u}\right\rangle\right]=\mu_{p}, \frac{1}{2}\left(\left[\left\langle P_{u}\right\rangle\right]^{2}-\left[\left\langle P_{u}\right\rangle\left\langle P_{u}\right\rangle\right]\right)=-\mu_{p}^{2}$ and $\operatorname{det}\left\langle P_{u}\right\rangle=-\mu_{p}^{3}$, the VEV matrix $\left\langle P_{u}\right\rangle$ must satisfy a cubic equation

$$
\left\langle P_{u}\right\rangle^{3}-\mu_{p}\left\langle P_{u}\right\rangle^{2}-\mu_{p}^{2}\left\langle P_{u}\right\rangle+\mu_{p}^{3} \mathbf{1}=0 .
$$

Therefore, we assume superpotential terms for $P_{u}$

$$
W_{P}=\lambda_{P}\left[\left(\Phi_{u} P_{u}-P_{u} \Phi_{u}\right) \Theta_{P}\right]+\varepsilon_{S B} \frac{\lambda_{P}^{\prime}}{\Lambda}\left[\left(P_{u} P_{u} P_{u}-\mu_{p} P_{u} P_{u}-\mu_{p}^{2} P_{u}+\mu_{p}^{3}\right) \Theta_{P}^{\prime}\right]
$$

Note that the second term in (3.3) cannot conserve the $\mathrm{U}(1)_{X}$ charges. (The superpotential terms (3.3) can conserve the $R$ symmetry, because the fields $\Theta_{P}$ and $\Theta_{P}^{\prime}$ are assigned as $R$ charges $R=2$, while $\Phi_{u}$ and $P_{u}$ are assigned as $R=0$.) We assume that such $\mathrm{U}(1)_{X}$ symmetry breaking terms are suppressed by a small factor $\varepsilon_{S B}$.

On the other hand, superpotential in the quark sector is given as follows:

$$
W_{q}=\mu_{u}^{X}\left[\Phi_{u} \Theta_{u}^{X}\right]+\mu_{d}^{X}\left[Y_{d} \Theta_{d}^{X}\right]+\sum_{q=u, d} \frac{\xi_{q}}{\Lambda}\left[\Phi_{e}\left(\Phi_{X q}+a_{q} E_{q}\right) \Phi_{e} \Theta_{q}^{X}\right]
$$

where the parameter $a_{u}$ is real, but $a_{d}$ is complex. Here the VEV matrices $\left\langle\Phi_{X q}\right\rangle_{e}$ and $\left\langle E_{q}\right\rangle_{e}$ are given by

$$
\left\langle\Phi_{X q}\right\rangle_{e} \propto X, \quad\left\langle E_{q}\right\rangle_{e} \propto \mathbf{1}
$$

and the factor $\left(\Phi_{X q}+a_{q} E_{q}\right)$ plays a role in breaking the $\mathrm{O}(3)$ flavor symmetry into a discrete symmetry $\mathrm{S}_{3}$ at the $e$ basis. If we regard $\Phi_{X q}$ and $E_{q}$ as $\Phi_{X u}=\Phi_{X d} \equiv \Phi_{X}$ and $E_{u}=E_{d} \equiv E$, we will obtain an unwelcome relation $Q_{X}\left(\Phi_{u}\right)=Q_{X}\left(Y_{d}\right)$. Therefore, we must distinguish $\left(\Phi_{X u}, E_{u}\right)$ from $\left(\Phi_{X d}, E_{d}\right)$. However, if we replace $\mu_{u}^{X}$ and $\mu_{d}^{X}$ in (3.4) with $\left[Y_{d}\right]$ and $\left[\Phi_{u}\right]$, respectively, we can regard $\Phi_{X q}$ and $E_{q}$ as $\Phi_{X u}=\Phi_{X d} \equiv \Phi_{X}$ and $E_{u}=E_{d} \equiv E$ without considering two sets $\left(\Phi_{X u}, E_{u}\right)$ and $\left(\Phi_{X d}, E_{d}\right)$. Superpotential terms for the field $\Phi_{X}$ are given by

$$
W_{X}=\varepsilon_{S B}\left\{\frac{\xi_{X}}{\Lambda}\left[\Phi_{X} \Phi_{X} \Phi_{X} \Theta_{X}\right]+\lambda_{X}\left[\Phi_{X} \Phi_{X} \Theta_{X}\right]\right\}
$$

with $\left(\lambda_{X} / \xi_{X}\right) \Lambda=-\left[\Phi_{X}\right]$, because

$$
[X]=1, \quad \frac{1}{2}\left([X]^{2}-[X X]\right)=0, \quad \operatorname{det} X=0 .
$$


Also, superpotential terms for the field $E$ are given by

$$
W_{E}=\varepsilon_{S B}\left(\mu_{E}\left[E \Theta_{E}\right]+\mu_{E}^{2}\left[\Theta_{E}\right]\right)
$$

Note that we cannot write the superpotential terms (3.6) and (3.8) without breaking the $\mathrm{U}(1)_{X}$ symmetry explicitly, so that we have added a factor $\varepsilon_{S B}$ in the superpotential terms (3.6) and (3.8).

In the superpotential terms (3.3), (3.6) and (3.8), we have assumed some specific forms of $\mathrm{U}(1)_{X}$ symmetry breaking terms. As such symmetry breaking terms, in general, not only those given in (3.3), (3.6) and (3.8) but also many other terms are allowed. Therefore, at present, the forms of the $\varepsilon_{S B}$-terms are only ones which are required from a phenomenological point of view.

\section{Concluding remarks}

In conclusion, for the purpose of explaining the observed tribimaximal neutrino mixing, we have proposed a yukawaon model (1.4) [(3.4)] in the quark sector, where the $\mathrm{O}(3)$ symmetry is broken into $\mathrm{S}_{3}$ by the $\operatorname{VEV}\left\langle\Phi_{X}\right\rangle_{e}$ on the $e$ basis (the diagonal basis of $\left\langle Y_{e}\right\rangle$ ). The up-quark mass matrix given in Eq.(1.4) includes one parameter $a_{u}$. In the seesaw-type neutrino mass matrix $M_{\nu}=m_{D} M_{R}^{-1} m_{D}$, the Majorana mass matrix of the right-handed neutrinos $M_{R} \propto\left\langle Y_{R}\right\rangle$ is related to the up-quark mass matrix $M_{u} \propto\left\langle Y_{u}\right\rangle \propto\left\langle\Phi_{u}\right\rangle\left\langle\Phi_{u}\right\rangle$ as given in Eq.(2.5) which includes one parameter $\xi$. The parameters $a_{u}$ and $\xi$ can describe 5 observables (2 up-quark mass ratios and 3 neutrino oscillation parameters). The numerical results for the neutrino oscillation parameters excellently gives nearly tribimaximal mixing as shown in Table 1.

So far, we did not mention neutrino mass ratios, i.e. $R=\Delta m_{\text {solar }}^{2} / \Delta m_{\text {atm }}^{2}$. The ratio $R$ can favorably be fitted by adding [1] a term $\lambda_{R}^{\prime}\left[Y_{\nu} Y_{\nu} \Theta_{R}\right]$ to the superpotential terms $W_{R}$ (3.7) [(3.1)], or by adding [10] a term $\left(y_{R}^{\prime} / \Lambda\right) \nu^{c} Y_{\nu} Y_{\nu} \nu^{c}$ to the would-be Yukawa interaction (1.7). Those terms play a role in shifting neutrino masses commonly without affecting neutrino mixing. However, the added parameter is exactly fixed by the observed value of $R$, so that there is no prediction in the neutrino sector.

Although the present model with (1.4) and (2.5) can describe values of 11 observables (4 quark mass ratios, 4 CKM mixing parameters, and 3 neutrino oscillation parameters) by adjusting 4 parameters ( 1 and 2 in the up- and down-quark sectors, respectively, and 1 in the $Y_{R}$ sector), the fitting for down-quark masses and the CKM mixing parameters is poor as seen in Table 2, although the results are qualitatively not so bad. The two parameter description in the down-quark sector is too tight. We think that the present model is a step in the right direction to a unified yukawaon model. In the next step, we will investigate a further improvement of the down-quark sector.

In the present model, we have assumed an $\mathrm{O}(3)$ flavor symmetry. The VEV relations which are derived from the superpotential under the $\mathrm{O}(3)$ symmetry are valid only in specific flavor bases which are connected by orthogonal transformations. We have regarded the $e$-basis (the diagonal basis of $\left.\left\langle Y_{e}\right\rangle\right)$ as a specific basis, in which the relations from the $\mathrm{O}(3)$ symmetry are valid and the VEV matrix $\left\langle\Phi_{X}\right\rangle$ takes a simple form (3.5).

The present approach based on a yukawaon model seems to provide a new view to a unified description of the masses and mixings differently from conventional mass matrix models. Under 
few parameters in the quark sector, the model can predict not only all quark and lepton mass ratios but also the CKM matrix parameters and neutrino oscillation parameters, although an improvement is still needed as far as the down-quark sector is concerned. It is worthwhile taking the present model seriously as a promising model which can give a unified description of the quark and lepton masses and mixings. Further development of the model is expected.

\section{Acknowledgments}

The author would like to thank T. Yamashita, M. Tanaka, N. Uekusa and H. Fusaoka

for helpful discussions. Especially, he thanks H. Fusaoka for pointing out incompleteness of the scenario in the earlier version. This work is supported by the Grant-in-Aid for Scientific Research (C), JSPS, (No.21540266).

\section{References}

[1] Y. Koide, Phys. Rev. D78 (2008) 093006 .

[2] Y. Koide, Phys. Rev. D79 (2009) 033009.

[3] Y. Koide, Phys. Lett. B665 (2008) 227.

[4] L. -L. Chau and W. -Y. Keung, Phys. Rev. Lett. 53, 1802 (1984); H. Fritzsch, Phys. Rev. D32 (1985) 3058.

[5] C. Amsler, et al., Particle Data Group, Phys. Lett. B667 (2008) 1.

[6] P. F. Harrison, D. H. Perkins and W. G. Scott, Phys. Lett. B458 (1999) 79; Phys. Lett. B530 (2002) 167; Z.-z. Xing, Phys. Lett. B533 (2002) 85; P. F. Harrison and W. G. Scott, Phys. Lett. B535 (2002) 163; Phys. Lett. B557 (2003)76; E. Ma, Phys. Rev. Lett. 90 (2003) 221802; C. I. Low and R. R. Volkas, Phys. Rev. D68 (2003) 033007.

[7] Y. Koide and H. Fusaoka, Z. Phys. C71 (1996) 459; Prog. Theor. Phys. 97, (1997) 459.

[8] C. D. Froggatt and H. B. Nielsen, Nucl. Phys. B 147 (1979) 277.

[9] Y. Koide, arXiv:0906.3370 [hrp-ph].

[10] Y. Koide, arXiv:0812.3203 [hep-ph], a talk at "Particle Physics, Astrophysics and Quantum Field Theory: 75 Years since Solvay" (PAQFT08), 27-29 Nov. 2008, Nanyang Executive Centre, Singapore, To appear in the Conference Proceedings (Intl.J.Mod.Phys.A).

[11] Z.-z. Xing, H. Zhang and S. Zhou, Phys. Rev. D77 (2008) 113016. Also, see H. Fusaoka and Y. Koide, Phys. Rev. D57 (1998) 3986.

[12] Y. Koide, Lett. Nuovo Cimento 34 (1982) 201; Phys. Lett. B120 (1983) 161; Phys. Rev. D28 (1983) 252. 
[13] D. G. Michael et al., MINOS collaboration, Phys. Rev. Lett. 97 (2006) 191801; J. Hosaka, et al., Super-Kamiokande collaboration, Phys. Rev. D74 (2006) 032002.

[14] B. Aharmim, et al., SNO collaboration, Phys. Rev. Lett. 101 (2008) 111301. Also, see S. Abe, et al., KamLAND collaboration, Phys. Rev. Lett. 100 (2008) 221803. 